\title{
LA ESCUELA ELEMENTAL DE ARTES E INDUSTRIAS DE BÉJAR
}

\author{
(1852-1902) \\ The Regional School of Arts and Crafts of Béjar (1852-1902) \\ Carmen CASCÓN MATAS \\ E-mail: ccasconm@hotmail.com
}

Fecha de recepción: 24-IX-2010

Fecha de aceptación: 28-I-2011

Resumen: La Escuela Regional de Artes y Oficios de Béjar (Salamanca) inició su andadura en 1886, aunque sus precedentes pueden ser rastreados desde 1852 en la Escuela Industrial. Béjar por aquel entonces podía presumir de ser uno de los escasos centros fabriles del oeste español, con una industria textil renqueante que subsistía a pesar de los embates de la crisis. La enseñanza técnica impartida en el centro educativo bejarano pretendía insertar a los trabajadores en el entorno laboral. El presente artículo intenta aportar una nueva perspectiva a la etapa comprendida entre 1886 y 1901 manejando nuevos datos procedentes del archivo de la propia institución, hasta ahora prácticamente inéditos, a la par que reflexionar sobre el contexto histórico, social y económico de la ciudad fabril.

Palabras clave: Escuela de Artes y Oficios, Béjar, Enseñanza Técnica, Industria Textil.

Abstract: The Regional School of Arts and Crafts of Béjar (Salamanca) began its activity in 1886, although its precedents can be traced from 1852 in the Industrial School. At that time Béjar could claim to be one of the few manufacturing centres in the west of Spain, with a limping textile industry wich survived despite the onslaughts of the crisis. The technical teaching given in the educational centre of Béjar tried to incorporate the workers in the labour environment. The present article tries to provide a new perspective to the period between 1886 and 1901 managing new data from the institution archives, unprecedented up to now, and to ponder about the historical, social and economical context of the manufacturing town at the same time.

Key Words: School of Arts and Crafts, Béjar, technical teaching, textile industry. 


\section{INTRODUCCIÓN}

Con este artículo pretendo dar a conocer la fase inicial de la Escuela Industrial (posteriormente Escuela de Artes y Oficios) comprendida entre 1852 y 1902. La primera fecha viene marcada por la fundación de la institución y la segunda por la inclusión del plan de los estudios técnicos superiores, aunque debido a sus avances y retrocesos, cierres, crisis y glorias, se pueden establecer otras periodizaciones internas fácilmente reconocibles. En nuestro caso hemos querido narrar en este texto únicamente la singladura de la formación técnica elemental, pues la enseñanza media o peritaje y la superior pertenecen ya a la etapa posterior a 1902.

Sin embargo, la Escuela no podría entenderse sin la necesidad acuciante de la alfabetización de las clases obreras, ni la voluntad de la burguesía por dotar a su industria de una mano de obra cualificada. Tampoco sin la participación de las instituciones municipales, diputación y estado en el proyecto. Porque el problema de la enseñanza no sólo era cosa de unos pocos, sino en general de toda una España que se industrializaba demasiado tarde y que debía mirar al exterior para encontrar ejemplos y modelos en los que mirarse.

Si la trayectoria económica de Béjar se hubiera estancado, si se hubiese quedado en un mero villorrio de paso, lejos de la capital, encaramada en las faldas de la sierra, viviendo apenas del comercio de arrieros que recorrían la Vía de la Plata, nunca se hubiese implantado la Escuela Industrial en 1852. Porque, en definitiva, la razón de su ser pivotaba en torno al eje económico de una manufactura textil que hacinaba a grupos antagónicos en un espacio mínimo, ahogados entre muros de granito, encerrados en sí mismos, sin apenas contacto con el exterior. La falta de comunicaciones favorecía el asilamiento y complicaba la industrialización. A pesar de todo, las fábricas medraban y según Pascual Madoz, que nos visitó allá por 1849, había en Béjar 200 fábricas que producían 754.600 varas de paño al año y daban empleo a 4.000 personas $^{1}$.

Las calles se hallaban pobladas de trabajadores sin rumbo, analfabetos, ávidos de un salario mínimo para subsistir. "Las costumbres de este pueblo se adulteran extraordinariamente. Como un gran centro manufacturero acuden a él los que no encuentran trabajo en otros pueblos de la Península y del extranjero. De esta inevitable aglomeración procede sin duda aquella adulteración, que motiva frecuentes asesinatos y continuas pendencias entre la juventud"2. A la vista de la situación para Nicomedes Martín Mateos la

1 SÁNCHEZ MARTÍN, J.R.: "Industria textil y fábricas en Béjar". Especial Béjar en Madrid, 30 de diciembre de 2005, p. 23.

2 CANO PAVÓN, J. M. "Las limitaciones de la enseñanza técnica obrera en la España Isabelina: la Escuela Industrial de Béjar (1852-1867)”. Lull. Palma de Mallorca, 24 (2001), p. 326. 
solución sólo podría centrarse en la instrucción pública, en la educación de las masas analfabetas. De ahí sus esfuerzos por expandir la enseñanza impulsando los estudios elementales, la educación técnica a través de la fundación de la Escuela Industrial y la cultura en general por medio de la creación del Círculo Obrero ${ }^{3}$. Incluso llegó a proponer al Ministerio la impartición de clases gratuitas de filosofía moral. El ayuntamiento la elevó al Ministerio y éste aceptó la propuesta ${ }^{4}$.

Mucha mano de obra, aunque escasamente preparada; una bomba de relojería a punto de explotar en cuanto a conflictividad social se refería. La burguesía industrial estaba al tanto de la situación y era la primera interesada en erradicar el analfabetismo. El pensamiento vendría a ser el siguiente: si se implanta una enseñanza con vistas a mejorar la preparación técnica de los trabajadores, obtendremos un doble progreso. El primero: descenderá la conflictividad social, disminuirán las huelgas y las fábricas producirán más. El segundo: obtendremos mano de obra cualificada aplicable a nuestras industrias y las fábricas producirán paños de mejor calidad ${ }^{5}$. En cuanto a la masa obrera general la implantación de la enseñanza técnica dará oportunidad a los vástagos de familias de posición media, sin posibilidad de costear una formación universitaria, a medrar y ocupar ciertos puestos con mayor nivel de cualificación. En definitiva significaba la potenciación de la industria textil bejarana, con una mejora de la cantidad y de la calidad de la producción, en medio de la paz social hipotética.

La teoría que manejaba una parte de la burguesía, sentada sobre estas bases, podemos decir que aspiraba a tener una solidez aparente. Sin embargo, bien es verdad que la alfabetización de las masas obreras podría llegar a tener un efecto totalmente contrario. Una vez adquiridos unos ciertos bagajes culturales, tales como saber leer y escribir, es claro que despertaba y aumentaba en los trabajadores una cierta conciencia social que podría provocar huelgas y demanda de condiciones de trabajo más favorables. De todo ello podemos deducir que no toda la burguesía vería con buenos ojos la fundación de la Escuela Industrial o la potenciación de la instrucción pública, cuyo máximo mentor era Nicomedes Martín Mateos. Quizás por ello, se le hizo responsable del fracaso de la primera fase de la enseñanza técnica en Béjar ${ }^{6}$.

3 HERNÁNDEZ DÍAZ, J.M.: La educación en la obra de Nicomedes Martín Mateos. Ed. Fundación Premysa, Béjar, 2007.

4 HERNÁNDEZ DÍAZ, J. M.: Educación y sociedad en Béjar durante el siglo XIX. Salamanca, Universidad de Salamanca, 1983, p. 217.

5 SÁNCHEZ MARTÍN, J.R.: “Industria textil y fábricas en Béjar”. Especial Béjar en Madrid, 30 de diciembre de 2005, p. 23.

6 CANO PAVÓN, J.M.: Ob. cit. Se cita en este artículo "el patrimonialismo" que ejercía Nicomedes en cuanto a la Escuela se refería, causas, que sumadas a otras, vendrían a 
Otra buena parte de la burguesía bejarana y de sus instituciones (Ayuntamiento, Sociedad Económica de Amigos del País, Junta de Fábrica) manifiestan un interés creciente por poner en marcha la Escuela Industrial, uniéndose esta última al devenir de la industria textil de tal forma, que se convertirá en su espoleta en tiempos de bonanza y en su panacea en periodos de crisis. Así, la primera fase del centro se verá enmarcada en un momento de prosperidad, mientras que en la segunda resurgirá como medio para salir de la crisis económica.

La Sociedad Económica de Amigos del País de Béjar se convertirá en una de las principales impulsoras de su fundación, con Nicomedes Martín Mateos a la cabeza. Sin embargo, esta particularidad no afecta sólo a Béjar, pues en otros lugares como Sevilla o Asturias (Oviedo) estas mismas sociedades serían también las promotoras de sus respectivas escuelas ${ }^{7}$.

\section{LAS ENSEÑANZAS TÉCNICAS EN ESPAÑA Y EN BÉJAR}

La burguesía española sólo tenía que mirar fuera como hemos dicho. La implantación de las enseñanzas técnicas es un proceso que surge en Europa, en el marco de la transición del Antiguo Régimen a la industrialización. En Francia se habían fundado la Escuela de Artes y Oficios en 1780 y el Conservatorio de Artes en 1828, modelo extendido después a otros países como Inglaterra e Italia ${ }^{8}$.

En lo que se refiere a España, su implantación tardará en llegar debido a las peculiaridades de su proceso industrializador, demasiado lento y regional. En lo que se refiere a la lentitud hay que tener en cuenta que en nuestro país la enseñanza industrial sólo se pondría en marcha a partir de la segunda mitad del siglo XIX, mientras que en Inglaterra, líder de la industria textil, funcionaba a pleno rendimiento desde finales del siglo XVIII ${ }^{9}$. En cuanto a su territorialidad, pensemos que España no se industrializó en su totalidad, sino que los centros modernizados plenamente se caracterizaban por su aislamiento y disparidad geográfica. Los núcleos industriales se situaban al norte (Asturias, País Vasco), este (Cataluña) y sur (Andalucía). Las enseñanzas técnicas no se pondrían en marcha hasta que la industrialización no conociera un cierto grado de desarrollo.

provocar el cierre de la misma.

7 DURÁN RODRÍGUEZ, Ma D.: "La educación técnica popular en Francia y España (1870-1950). Algunas consideraciones de las Escuelas de Artes y Oficios en ambos países". En Sarmiento. Anuario Gallego de Historia de la Educación, n 13, 2009, pp. 80-81.

8 Para conocer más sobre el tema consultar Ibídem, pp. 69-101.

9 Ibídem, p. 74. 
En lo que se refiere a los apoyos sociales, según José María Hernández Díaz "la erección y sostenimiento de las Escuelas de Artes y Oficios en la España de la Restauración es el resultado del apoyo prestado por diversas iniciativas populares, privadas, eclesiásticas, municipales o de la Administración central (en mucho menor grado ésta) que intervienen en el tema de manera muy desigual" 10 .

Aun con todo, y según las palabras de $\mathrm{M}^{\mathrm{a}}$ Dolores Durán, se apreciaba el "desdén con el que la sociedad de entonces, tanto en España como en otros países de nuestro entorno, veía los estudios técnicos, a los que se acudía por su utilidad pero que se dejaban para los hijos de las clases populares, obreras y pequeño burguesas, reservando otros estudios socialmente más prestigiosos, como los universitarios o los de las grandes escuelas, para los hijos de las clases privilegiadas"11. Cada región implantará una escuela adaptada a sus necesidades industriales o comerciales, variando su oferta educativa según esta premisa. En el caso que nos ocupa es claro que las asignaturas pivotaban hacia una especialización textil, destacando la asignatura de Taller de Tejidos.

En cuanto a los orígenes de la enseñanza técnica en Béjar, ya en 1825 el ayuntamiento daba licencia al tintorero francés Miguel Faure, oficial de Superintendente y Director de las Reales Fábricas de Guadalajara y Brihuega, para que instruyera a los fabricantes, aunque sin crear un centro docente adecuado a la impartición de clases magistrales ${ }^{12}$. En 1835 el conde de las Navas Luis Pizarro Ramírez, diputado a Cortes y miembro de la Sociedad Económica de Amigos del País de Béjar, apeló ante la reina Isabel II a favor de la necesidad de mano de obra especializada y el asentamiento de técnicos extranjeros, así como la creación de dos cátedras, una de matemáticas y otra de física y química para tal fin ${ }^{13}$. Sin embargo, la falta de medios y voluntad impidió la realización de estos proyectos.

\section{La ESCUELA FrACASADA (EsCuela Industrial, 1852-1868)}

Podemos llamar primer periodo de la Escuela Industrial de Béjar a la comprendida entre 1852 y 1867. Su nacimiento, surgido de la voluntad aunada del ayuntamiento y de la burguesía local, vendría a confirmar el

10 HERNÁNDEZ DÍAZ, J.M.: "Burguesía liberal y educación popular en la España interior. Las Escuelas de Artes y Oficios en la Restauración”, en Clases populares, cultura, educación. Ss. XIX y XX, Coloquio hispano-francés. Casa de Velázquez - UNED. Madrid, 1990, pp. 241-251.

11 DURÁN RODRÍGUEZ, M.D.: Ob. Cit, p. 77.

12 HERNÁNDEZ DÍAZ, J. M.: Educación y sociedad en Béjar durante el siglo XIX. Salamanca, Universidad de Salamanca, 1983, p. 202.

13 Ibídem., p. 205. 
reconocimiento del estado a la importancia fabril del centro bejarano frente a sus competidores catalanes.

La medida que dinamitaría la situación de estancamiento en que se sumía el proyecto educativo bejarano fue la nueva legislación surgida en 1850. Esta permitía la creación de escuelas elementales con la aportación económica de ayuntamiento, diputación y gobierno. El 20 de julio de 1852, y mediante real orden, se autorizó la apertura de la Escuela Industrial de Béjar con un presupuesto inicial de 48.000 reales $^{14}$, proceso en el cual tuvo mucho que ver el ministro de Fomento Mariano Miguel de Reinoso.

La Escuela Industrial ofrecería una formación técnica elemental con horario nocturno, contando con un profesor para el curso preparatorio (impartido por José García), tres catedráticos (dos de matemáticas y uno de física, química y mecánica), un ayudante y un mozo, además de los estipendios extras a pagar al profesor que ejerciera como secretario y el que impartiera las clases de dibujo y modelado ${ }^{15}$. El primer director de la Escuela y profesor de matemáticas, Nicomedes Martín Mateos ${ }^{16}$, fue el encargado de pronunciar el discurso inaugural el día 15 de noviembre de $1852^{17}$ en el primer local de la misma, un edificio compartido con la Escuela Municipal de Enseñanza Elemental situado en la calle Mansilla. Posteriormente la Escuela Industrial se trasladaría a otro edificio de esta misma calle con entrada por Quebradilla de San Francisco en septiembre de $1853^{18}$. Por último, en 1866, dos años antes de su desaparición, el consistorio decidió instalarla en el edificio del viejo Palacio Ducal. En la sesión de 28 de agosto de ese año, el ayuntamiento contestó a una carta enviada por el Rector de la Universidad de Salamanca a este respecto, señalando que se había procedido de esta forma porque no tenían dinero suficiente para el alquiler del anterior local y que el pago del arrendamiento al administrador del duque por 4 habitaciones y dos cuartos para el material era suficiente para el desempeño correcto de la enseñanza ${ }^{19}$.

En general, la corta de vida de esta primera Escuela estuvo marcada

14 "Memoria descriptiva correspondiente a los años 1947-1952 y conmemorativa del Primer Centenario de la Escuela" Escuela de Peritos Industriales y Elemental de Trabajo de Béjar. s/f

15 CANO PAVÓN, J.M: ob cit, p. 325.

16 Para conocer más sobre este personaje recomendamos HERNÁNDEZ, DÍAZ, J. Ma Don Nicomedes Martín Mateos. Antología de textos breves. Ed. Casino Obrero de Béjar y Caja de Ahorros de Salamanca. Enero de 1990.

17 HERNÁNDEZ DÍAZ, J.M.: "Filosofía, ciencia y tecnología en el discurso de don Nicomedes Martín Mateos al inaugurar la Escuela Industrial de Béjar (1852)". Revista nº 6, del Centro de Estudios Bejaranos, diciembre de 2002. Ed. CEB y Ayuntamiento de Béjar.

18 Archivo Municipal de Béjar: Libros de actas de sesiones. Sesión de 23 de septiembre de 1853.

19 Archivo Municipal de Béjar: Libros de actas de sesiones. Sesión de 28 de agosto de 1866. 
por las escasas subvenciones, la limitación del espacio con el que se contaba, la casi total ausencia de material $\operatorname{escolar}^{20}$ y el parco número de profesores con bajos salarios. En una situación así era prácticamente imposible que la Escuela pasara de ser un centro circunscrito al nivel elemental. Al llegar al tercer año de vida, y con el fin de adaptarse a la normativa vigente, se hizo necesario contratar más personal. De esta forma se sumó a la plantilla, que contaba ya con la presencia de Nicomedes Martín Mateos (director y profesor de matemáticas), José García (en 1858 sustituido por Gregorio Laso de la Vega en el curso preparatorio), Victoriano Díez (dibujo) y Primo Comendador (ayudante interino de geometría, física y química), Bernardo Cañizares (física y química) ${ }^{21}$.

Sin embargo, las dificultades no hicieron más que acrecentarse. Además de las carencias que arrastraba desde su fundación, habrá que añadir dos nuevos problemas que surgieron al calor de la nueva legislación de 1857 1858. Por un lado, se exigía la existencia de un instituto, del que Béjar carecía, para alojar las enseñanzas preparatorias, que se escindían de esta forma de las Escuelas Industriales ${ }^{22}$. Por otro, se imponía como norma el previo pago de la matrícula y la inscripción de los alumnos en Salamanca. Dado que la mayoría de los estudiantes se encontraban en el segmento social de obreros sin recursos, la Escuela hubiera puesto punto y final a su trayectoria en ese momento, pero el gobierno mantuvo el plan docente excepcionalmente en Béjar por petición municipal ${ }^{23}$.

Entre 1861 y 1864, el rector de la Universidad de Salamanca, Tomás Belestá hizo dos inspecciones y escribió sendos informes sobre el estado del centro. En ellos destaca la pobreza general de la institución, la pésima ordenación del material científico, la ausencia del pago de matrícula por los alumnos, el aumento de las enseñanzas primarias, el inmovilismo de la plantilla pues seguía a su frente como director Nicomedes Martín Mateos ${ }^{24}$. A partir de ese momento, la Escuela Industrial sufrirá un acelerado declive agudizado por el cese de éste como director en 1865 (sustituido por Primo Comendador Téllez). En 1868 las instalaciones cierran sus puertas ante la suspensión total de la subvención por parte del ayuntamiento, centrado en la creación del Instituto Municipal de Segunda Enseñanza ${ }^{25}$.

20 CANO PAVÓN, J.M.: ob cit. Sobre los materiales docentes y las subvenciones, pp. 336-337.

21 Ibídem, p. 328. Para conocer la situación del profesorado, p. 333-336.

22 Ibídem, p. 329.

23 Ibídem, p. 329.

24 Ibídem, p. 330.

25 HERNÁNDEZ DÍAZ, J. M.: Educación y sociedad en Béjar durante el siglo XIX. Salamanca, Universidad de Salamanca, 1983, p. 212. 
La ESCUEla Renovada (Escuela municipal de Artes y Oficios, 1879-1886)

En el segundo periodo de la Escuela de enseñanza técnica elemental, ahora llamada Escuela de Artes y Oficios, podemos distinguir dos fases: la comprendida entre 1879 y 1886, y la de 1887 y 1902, año en que implanta la enseñanza media ${ }^{26}$.

Después del parón producido en 1868, resurge la Escuela Técnica de Béjar en 1879, mucho antes que en otros lugares de la provincia (véase el ejemplo de Ciudad Rodrigo, Peñaranda, Alba de Tormes y la misma Salamanca) a iniciativa de una resurgida Sociedad Económica de Amigos del País ${ }^{27}$. La voluntad de los intelectuales y de una parte de la burguesía va por delante de los proyectos oficiales, incluso al margen de estos. La misión de la Escuela será la de mantener a flote una industria en plena crisis tras el cierre definitivo del conflicto carlista ${ }^{28}$. En efecto, el estado había firmado hasta entonces contratas con las fábricas bejaranas para los uniformes militares, como ya lo venía haciendo desde $1715^{29}$. Sin embargo, con la paz definitiva, las perspectivas no eran muy halagüeñas. La dependencia excesiva del textil bejarano con respecto al estado y su excesiva especialización en este campo sería, a la postre, una de las causas de su declive.

Esta nueva enseñanza industrial posee elementos de continuidad con respecto a la anterior Escuela, junto con otros nuevos que hacen que, al contrario que en la experiencia previa, este centro educativo acabe prosperando.

La continuación viene marcada por dos motivos. Por un lado, la Escuela de Artes y Oficios surge, como la Industrial, al margen de la iniciativa estatal sostenida únicamente por el ayuntamiento y la Sociedad Económica, una de las causas del fracaso de 1868. En la reseña de la inauguración recogida en el periódico La Locomotora se apuntaba que su andadura se había iniciado "con escasísimos recursos sí, pero con una fe y un entusiasmo que fue siempre creciendo a medida que aumentaban las dificultades inherentes a toda nueva creación en el principio de su desarrollo"30. La falta de capital

26 Ibídem, p. 222.

27 Ibídem, p. 222.

28 Ibídem, p. 224.

29 SÁNCHEZ MARTÍN, J.R.: "Industria textil y fábricas en Béjar" Especial Béjar en Madrid, 30 de diciembre de 2005, p. 22. Los inicios de la relación textil entre Béjar (centro productor) y el Estado (cliente) comenzó en 1715 a la llegada de los Borbones en detrimento de los centros productores catalanes por el apoyo de estos últimos a la Casa de Austria en la Guerra de Sucesión.

30 Recogida su transcripción completa en HERNANDEZ DÍAZ: Educación y sociedad en Béjar durante el siglo XIX. Salamanca, Universidad de Salamanca, 1983, pp. 225-226. 
ensombrecía de nuevo una institución que no pasaba del nivel elemental. Por otro lado, los artífices del proyecto vendrían a ser prácticamente los mismos (ayuntamiento y fabricantes junto con intelectuales) e incluso se eligió como nuevo director a Primo Comendador que ya lo había sido de la anterior institución.

Pero del fracaso se extrae un aprendizaje: el ayuntamiento trabajó entre 1879 y 1886 para que la Escuela de Béjar fuera reconocida oficialmente y subvencionada por el estado, hecho que se produjo en 1886 por Real Decreto. De esta forma, la de nuestra ciudad sería la única que obtendrá este reconocimiento en la provincia de Salamanca ${ }^{31}$.

\section{La Escuela institucionalizada (Escuela Regional de Artes y} Oficios, 1887-1900)

Desde este momento la Escuela, denominada ahora de Artes y Oficios, se integrará en un plan de apoyo a las escuelas técnicas elementales fomentado por el estado y repartido por la geografía nacional.

La ceremonia de inauguración se celebró el 14 de noviembre de 1887 (creada oficialmente el 20 de octubre de ese mismo año) en el edificio de la calle Mansilla y a ella asistieron autoridades civiles y militares, diputado a Cortes Jerónimo Rodríguez Yagüe, Sociedad Económica de Amigos del País, Junta de Fábrica y miembros de la suprimida Escuela Municipal de Artes y Oficios. El discurso de apertura fue pronunciado por Luis Caballero Noguerol y en él hizo hincapié de "la utilidad que reportan las Escuelas de Artes y Oficios en poblaciones industriales como la nuestra y apropósito de la crisis por que atraviesa la industria lanera (...) hizo atinadas observaciones a la marcha lánguida que en ésta se viene sumiendo desde hace unos años a esta parte e indicó los medios que a su juicio debieran ponerse en práctica por fabricantes y obreros para conjurar la crisis que amenaza con terminar con nuestra industria" 32 .

Las asignaturas impartidas eran "Aritmética, Geometría y principios de la construcción", "Dibujo geométrico industrial", "Física, química y mecánica", "Dibujo de adorno y figura", "Modelado y vaciado" y "Taller de Tejidos", ésta última surgida a partir de $1889^{33}$.

La plantilla en sus inicios se componía de un número de tres profesores (Primo Comendador Téllez, que además siguió desempeñando el cargo de

31 Ibídem, p. 227.

32 Archivo del I. E. S. "Río Cuerpo de Hombre": Libro de actas de sesiones de la Escuela Regional de Artes y Oficios, f. 3.

33 Se puede rastrear en el libro antedicho las asignaturas, profesores, número de alumnos y demás datos que se manejan sobre la Escuela durante esta etapa. 
director, Luis Caballero Noguerol y José Illán Manzanares), tres ayudantes (Evaristo Nieto Gómez, José Villaamil, Ramón Martín Bonisana y Enrique Brochín Comendador), un escribiente, un conserje y dos mozos de aseo.

El entusiasmo del que hablábamos (uno de los pilares imprescindibles para el éxito de la Escuela de Artes y Oficios) debía repartirse entre las instituciones, encargadas de la obtención del capital necesario para sobrevivir, y la sociedad bejarana en general. En este sentido, además del ayuntamiento, hay que destacar las aportaciones anuales de la Junta de Fábrica y de la Sociedad Económica de Amigos del País en forma de becas y premios a los alumnos con mejores expedientes. Por ejemplo, en la ceremonia de inauguración oficial del curso 1887-1888 se repartieron 40 pesetas por la Junta de Fábrica al estudiante más aventajado en Taller de Tejidos, 25 pts. por la Sociedad Económica de Amigos del País y otras compensaciones por el consistorio bejarano ${ }^{34}$. Posteriormente se sumó a esta política de gratificaciones el Círculo Obrero con 20 pts al mejor trabajo en Dibujo Geométrico Industrial ${ }^{35}$.

En cuanto al presupuesto, según los datos de que disponemos, hacia 1888 ascendía a un total de 24.375 pesetas, repartiéndose entre personal $\left(22.375\right.$ pts) y material $\left(12.000\right.$ pts) ${ }^{36}$. El número de alumnos varió a lo largo de esta etapa. En 1888 se permitía la matriculación de 282 personas $^{37}$, aunque en ese año sólo se registraron 203. Las clases seguían impartiéndose en horario nocturno.

Desde los inicios de esta etapa se atisba un deseo generalizado por mejorar, por impartir una docencia más completa y eficaz. En la sesión de profesores celebrada el 20 de Febrero de 1888 se aprobaba la petición a la superioridad de la instalación de un taller y un laboratorio de tejidos con aplicación al aprendizaje del tintado de paños ${ }^{38}$. Dada la escasez del espacio disponible en el viejo caserón de la calle Mansilla, se llegó a proponer al consistorio adecuar "un alto desván de la parte norte del edificio que ocupa esta escuela y que está en estado ruinoso" ${ }^{39}$. El ayuntamiento aprobó la propuesta, convirtiéndose además en el sostén económico de la nueva asignatura, Taller de Tejidos, con duración de tres años para la obtención del título y desembolso anual de 1.750 pts destinadas al pago del profesor.

34 Ibídem, inaugural de 8 de noviembre de 1887, f. 4.

35 Ibídem, 27 de mayo de 1888, f. 12.

36 Ibídem, 17 de enero de 1888, f. 6.

37 Ibídem, 15 de septiembre de 1888, f.1. En concreto 100 en Aritmética, Geometría y principios de construcción; 100 en nociones de Física, Química y Mecánica; 32 en Dibujo Geométrico Industrial; 38 en Dibujo de Adorno y Figura; y 12 en Modelado y Vaciado.

38 Ibídem, 20 de febrero de 1888, f. 8.

39 Ibídem, 21 de octubre de 1888, f. 18. 
Según declaración del profesorado era necesario "dotar a esta enseñanza del material necesario, dado los adelantos modernos, manifestarles la necesidad de una supervención que la coloque a la altura que le corresponde dada su importancia" 40 . En su apoyo definitivo a la institución tuvo mucho que ver el éxito obtenido por la Escuela en los muestrarios y dibujos de tejidos remitidos a la Exposición Universal de Barcelona, ya que se alzaron con dos premios: Medalla de bronce y mención honorífica ${ }^{41}$. El primer profesor de la misma fue Lorenzo Mira.

Por otro lado, se hacían patentes las deficiencias en cuanto a material docente, reducido espacio o malas condiciones del edificio. Luis Caballero Noguerol se quejó de "que la Cátedra de Dibujo Geométrico, que le está encomendada, se hallaba en muy malas condiciones, tanto por hallarse un local sumamente reducido, dos clases numerosas, ésta y la de Dibujo de Adorno y Figura, con lo que padecía la disciplina que debiera haber en unas y otras, porque las tres mesas en donde trabajaban sus alumnos eran inadecuadas por completo e insuficiente el alumbrado ${ }^{42}$ ". La Escuela pidió así al ayuntamiento tanto la ampliación del local como la subvención necesaria para el material ${ }^{43}$.

En cuanto a la ampliación del local se refiere, la solución tardaría en llegar. En 1888 las declaraciones dejadas por escrito en la sesión de la junta de profesores pueden describirse como alarmantes, pues en la carta dirigida a la superioridad se dice que "habiendo observado que ceden las vigas del techo de la clase de Física y teniendo, por tanto, que pueda ocurrir algún hundimiento, me veo en la necesidad de comunicarlo a Vuestra Excelencia a fin de que se sirva dar las consabidas órdenes oportunas para que sean reconocidas lo más pronto posible" ${ }^{44}$. De nuevo, en 1893 se vuelve insistir sobre la deficiencia de las instalaciones, definiendo como "ruinoso" el edificio que ocupaban y rogando la puesta en marcha de las obras necesarias para mejorar "la seguridad personal", "la higiene y la cultura de la población y la enseñanza pública" 45 . Durante el verano de ese año se llevaron a cabo

40 Archivo del I.E.S. "Río Cuerpo de Hombre": Libro copiador de la correspondencia dirigida a la superioridad (1888-1890). Carta de 23 de marzo de 1889, f. 45.

41 El caso se ha tratado en CASCÓN MATAS, M ${ }^{a}$ C: "Béjar y la Exposición Universal de Barcelona”. Béjar en Madrid, n 4.610. 23 de julio de 2010.

42 Archivo del I.E.S. "Río Cuerpo de Hombre": Libro de actas de sesiones de la Escuela Regional de Artes y Oficios, 29 de septiembre de 1890, f. 32.

43 Ibídem, 29 de mayo de 1891, ff. 36 y 37.

44 Archivo del I.E.S. Río Cuerpo de Hombre: Libro de correspondencia dirigida a la superioridad, 20 de diciembre de 1888, f. 42.

45 Archivo del I.E.S. "Río Cuerpo de Hombre": Libro de actas de sesiones de la Escuela Regional de Artes y Oficios Ibídem, 31 de diciembre de 1893, ff. 57 y 58. También en Libro de 
reparaciones, aunque no sería hasta 1899, y gracias a la insistencia de Marcelino Cagigal Valdés ${ }^{46}$ (profesor numerario de Aritmética, Geometría y principios de la construcción desde septiembre de $1898^{47}$ ), cuando la alcaldía ceda el espacio de la antigua parroquia de San Gil (posteriormente hospital, casa-cuna y Biblioteca Popular) a la Escuela.

Hemos apreciado un error en casi todas las monografías y artículos dedicados al estudio de esta institución al situar los locales en San Gil desde 1880 e incluso afirmar que ésta se trasladó por completo allí. Sin embargo, la documentación deja claro que sólo se cede esta construcción a la Escuela de Artes y Oficios en 1897 para la instalación de una sola de sus asignaturas, mientras que las restantes se siguen impartiendo en el antiguo edificio de la calle Mansilla ${ }^{48}$. De hecho los nuevos locales fueron destinados a la asignatura de Aritmética y Geometría, utilizándose para la solemne ceremonia de apertura del curso de 1899-1890.

En ella también se estrenaría el alumbrado eléctrico de la Escuela. El proceso de sustitución del alumbrado de petróleo tardó en llegar por la escasa potencia que ofrecían las compañías eléctricas asentadas en Béjar. Así, el cambio no sería posible hasta la fundación de la Sociedad de "La Abeja", compañía con la que la Escuela contrató el suministro ${ }^{49}$ de la clase instalada en el espacio de San Gil. El edificio de la calle Mansilla continuó con el alumbrado de petróleo.

El proyecto de instalación de un telar mecánico para ampliar las enseñanzas prácticas de la asignatura de Taller de Tejidos (petición realizada al ayuntamiento en 1891) también se demoró en demasía. El consistorio se negó a pagar el proyecto y la Escuela se vio en la necesidad de reunir

correspondencia dirigida a la superioridad, 24 de abril de 1894, f. 124. En este último se define perfectamente en estado del edificio de Mansilla "local pequeño, mezquino, insuficiente y por añadidura ruinoso en su mayor parte".

46 Archivo del I.E.S. "Río Cuerpo de Hombre": Libro de actas de sesiones de la Escuela Regional de Artes y Oficios, 19 de octubre de 1898, ff. 114 y 115.

47 Para conocer más sobre este personaje SÁNCHEZ MARTÍN, J.R.: "Correspondencia de Cagigal a Unamuno. Primera parte: de 1902 a 1920". Revista del Centro de Estudios Bejaranos, no 13. Diciembre de 2009. Ed. CEB y Ayuntamiento de Béjar, pp.153-170.

48 Ibídem, 12 de enero de 1899, f. 119. "Se dio lectura a otra comunicación del Sr. Alcalde cediendo a la Escuela la que fue Ermita de San Gil y antigua Casa-Cuna, para dar amplitud a las deficiencias del local de la Escuela y dedicarlas a las necesidades de la enseñanza". También en el mismo archivo Comunicaciones dirigidas a la Superioridad, f. 179. Carta del Director al Ayuntamiento de Béjar solicitando la amplitud del local y el cese de la CasaCuna.

49 Archivo del I.E.S. "Río Cuerpo de Hombre": Libro de actas de sesiones de la Escuela Regional de Artesy Oficios, 30 de septiembre de 1899, f. 128 
pacientemente la cantidad precisa para adquirir la maquinaria. Además, la idea no podía ponerse en marcha sin el suficiente espacio, problema que, como ya hemos visto, no se solventó hasta 1897. Sin embargo, mucho antes, ya en 1891 se solicitaba al ayuntamiento la ampliación del local por la compra de un motor de vapor ${ }^{50}$, en 1892 se adquiriría un motor de petróleo de fuerza 3 caballos $^{51}$ y en 1895 un telar mecánico a la empresa de telares de Sabadell Cañameras ${ }^{52}$. Una vez traslada la asignatura de Aritmética y Geometría a San Gil, el taller de Tejidos gozaría de mayor espacio para la instalación de toda la maquinaria.

También es reseñable hacer constar las evidencias de la delincuencia en la sociedad bejarana a las que se alude directamente en los libros pertenecientes a este periodo de la Escuela de Artes y Oficios, uno de los males que se quería atajar con la expansión de la enseñanza entre las clases popular y uno de los caballos de batalla de don Nicomedes Martín Mateos. Efectivamente, en una carta dirigida al consistorio con fecha 14 de noviembre de 1896 se dice que "el Profesor de la asignatura de Aritmética y Geometría se esta Escuela de Artes y Oficios se ha visto obligado esta noche a suspender la clase poco después de empezarla, por causas del escándalo que en la vía pública, y por bajo de las ventanas de dicha aula, producían varios jóvenes, y como esos escándalos, aunque en menor escala, viene ocurriendo casi a diario", por lo que se exigía una mayor vigilancia policial ${ }^{53}$.

En 1900, y por Real Orden, la anterior Escuela Regional de Artes y Oficios mudó su nombre por el de Escuela de Artes e Industrias, estando la de Béjar bajo la dirección de Marcelino Cagigal Valdés. Sin embargo, la verdadera reforma sobrevendría dos años más tarde.

\section{La Escuela Elemental y la Escuela Superior de Industrias} (Escuela De Artes E Industrias, 1900-)

Por Real Decreto de 17 de agosto de 1901, se crean las nueve Escuelas Superiores de Artes e Industrias en España (lo que podríamos llamar enseñanza media o Peritaje Industrial), una de las cuales sería la de Béjar (junto con la de Madrid, Alcoy, Gijón, Cartagena, Las Palmas, Tarrasa, Vigo y Villanueva y la Geltru). De esta forma se separarían la Escuela Elemental y la Superior, siendo voluntad del estado la implantación en Béjar de esta

50 Archivo del I.E.S. Río Cuerpo de Hombre: Libro de correspondencia dirigida a la superioridad, 14 de noviembre de 1896, f. 79.

51 Archivo del I.E.S. "Río Cuerpo de Hombre": Libro de actas de sesiones de la Escuela Regional de Artesy Oficios, 31 de agosto de 1892, f. 46.

52 Ibídem, 19 de junio de 1895, f. 72.

53 Archivo del I.E.S. Río Cuerpo de Hombre: Libro de correspondencia dirigida a la superioridad, 14 de noviembre de 1896, f. 150. 
última y la supresión de la primera. Los profesores, sin embargo, deciden instar a la superioridad a fin de mantener ambas enseñanzas ${ }^{54}$ por medio de las gestiones del diputado Nicolás Oliva (que sería nombrado Comisario Regio y Director de la Escuela) ante el ministro conde de Romanones. De hecho en julio de 1902 se convocó una manifestación pacífica desde la Escuela para que esta medida se llevara a efecto ${ }^{55}$, siendo aceptada la propuesta por el estado. De nuevo por voluntad de los bejaranos la Escuela salía adelante, siempre atenta al futuro y al desarrollo de la industria textil.

Para su implantación fue necesario el nombramiento de nuevos profesores, la adquisición de materiales para los talleres y la implantación de un mayor número de asignaturas como francés, contabilidad, geografía de España o lengua castellana ${ }^{56}$.

El mismo conde de Romanones asistió a la apertura del curso que contó también con la presencia del Rector de la Universidad de Salamanca, Miguel de Unamuno.

En 1903 la Escuela Superior de Industrias se trasladaría al edificio del antiguo Convento de San Francisco, donde permanecerá hasta 1948. La inauguración del curso 1903-1904 contaría de nuevo con la presencia de Miguel de Unamuno, quien pronunciaría el discurso de apertura de éste y de los cursos 1907-1908, 1908-1910 y 1910-1911 .

Pero, ésta ya es otra historia.

\section{Agradecimientos:}

Me gustaría agradecer a la directora del I.E.S. "Río Cuerpo de Hombre" el que me haya facilitado el acceso a los archivos del centro, en especial a Manuel Casadiego y a Juan Hernández, así como a Javier Ramón Sánchez Martín.

54 Ibídem, 19 de julio de 1902, f. 183.

55 Ibídem, 23 de Julio de 1902, f. 186.

56 Ibídem, 30 de septiembre de 1902, f. 189.

57 REDONDO QUINTELA, F: Unamuno y la Escuela Superior de Industrias de Béjar. Ed. Revide, Béjar 1996. 